\title{
Growth and magnetic properties of thin epitaxial $\mathrm{Ni}_{x} \mathrm{Pd}_{1-x}$ alloy films on $\mathrm{Cu}_{3} \mathrm{Au}(100)$
}

\author{
M. Seider, R. Kaltofen, and U. Muschiol, ${ }^{\text {a) }}$ \\ Institut für Festkörper- und Werkstofforschung (IFW) Dresden, Helmholtzstraße 20, D-01069 Dresden, \\ Germany \\ M.-T. Lin \\ Department of Physics, National Taiwan University, 106 Taipei, Taiwan \\ C. M. Schneider \\ Institut für Festkörper- und Werkstofforschung (IFW) Dresden, Helmholtzstraße 20, D-01069 Dresden, \\ Germany
}

We investigated the magnetic properties of $\mathrm{Ni}_{x} \mathrm{Pd}_{1-x}$ films epitaxially grown on $\mathrm{Cu}_{3} \mathrm{Au}(100)$ surfaces. The films grow nicely layer-by-layer at higher Pd concentrations and exhibit a composition dependent inverse spin reorientation transition. Growth and magnetic properties are found to be strongly affected by the film strain. (C) 2000 American Institute of Physics.

[S0021-8979(00)84108-0]

Complex magnetic alloys and compounds currently receive great technical interest because of their applications in mass storage technology and magnetoelectronics. Although the magnetic properties of binary and ternary systems have been extensively studied on bulk samples, ${ }^{1}$ only limited knowledge is available for ultrathin layers and particularly epitaxial films of these materials. The latter represent welldefined model systems and allow the study of the fundamental physical mechanisms governing the magnetism in alloys and compounds. Of specific relevance are the interrelations between crystalline structure, chemical composition, and electronic structure which in turn determine the magnetic behavior of the films. This knowledge is mandatory in order to be able to tailor magnetic materials with novel properties.

Recently a number of studies have been devoted to epitaxial films of binary ferromagnetic alloys involving various combinations of $3 d$ transition metals, e.g., $\mathrm{Fe}_{x} \mathrm{Ni}_{1-x}$, $\mathrm{Fe}_{x} \mathrm{Co}_{1-x}$, and $\mathrm{Co}_{x} \mathrm{Ni}_{1-x} \cdot{ }^{2-6}$ They revealed novel phenomena which cannot be observed in elemental films, such as new magnetic phases ${ }^{5}$ and composition-dependent spinreorientation transitions (SRT). ${ }^{6}$ On the other hand, alloys between $3 d$ and $5 d$ materials, e.g., $\mathrm{Co}_{x} \mathrm{Pt}_{1-x}$, exhibit unique magneto-optical properties introduced by the higher spinorbit coupling of the $5 d$ element. $^{7}$

In our study we concentrated on ultrathin $\mathrm{Ni}_{x} \mathrm{Pd}_{1-x}$ films which were epitaxially grown on $\mathrm{Cu}_{3} \mathrm{Au}(100)$ surfaces. In the bulk, the NiPd alloy assumes an fcc structure and is known to exhibit ferromagnetic ordering down to a $\mathrm{Ni}$ content as low as $3 \% .{ }^{8}$ One may therefore expect that films can be stabilized over a large compositional range without encountering structural changes or abrupt variations of the magnetic moment. The pure Ni films on $\mathrm{Cu}_{3} \mathrm{Au}(100)$ exhibit an inverse SRT, similar to the one observed in $\mathrm{Ni} / \mathrm{Cu}(100){ }^{9,10}$ In this case the magnetization turns from inplane to out-of-plane with increasing film thickness. A simi-

\footnotetext{
a)Electronic mail: u.muschiol@ifw-dresden.de
}

lar phenomenon has not yet been observed in alloy films.

The experiments were carried out in a standard UHVchamber with a base pressure better than $2 \times 10^{-10}$ mbar. During film growth the pressure could be kept below 5 $\times 10^{-10}$ mbar. The $\mathrm{Cu}_{3} \mathrm{Au}(100)$ single crystal surface was cleaned by Ar ion bombardment, followed by flashing the sample up to $780 \mathrm{~K}$. A subsequent annealing for half an hour at $600 \mathrm{~K}$ helped to restore the chemical order of the template. The films were codeposited from individual $\mathrm{Ni}$ and $\mathrm{Pd}$ electron beam sublimators with the sample held at $T=300 \mathrm{~K}$. The growth was monitored in situ by recording the intensity of the specularly diffracted beam in medium energy electron diffraction (MEED). This also served as a precise measure of the film thickness. Knowing the film thickness, the composition of the films was determined from Auger electron spectroscopy.

In Fig. 1 we compare the growth of NiPd alloy films

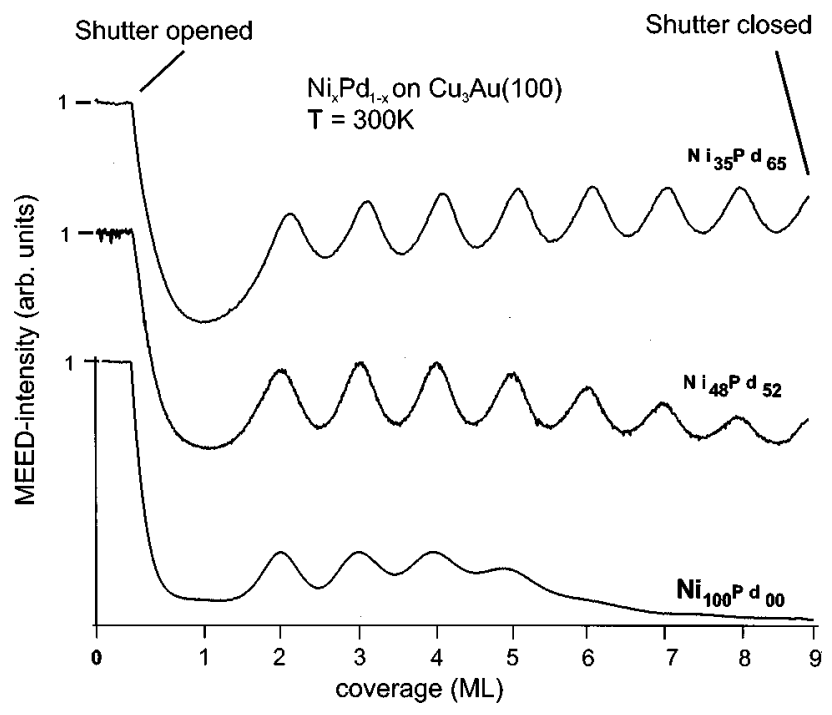

FIG. 1. The MEED oscillations during the growth of pure $\mathrm{Ni}$ and $\mathrm{Ni}_{x} \mathrm{Pd}_{1-x}$ alloy films. 
a)

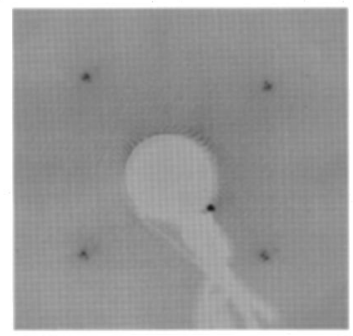

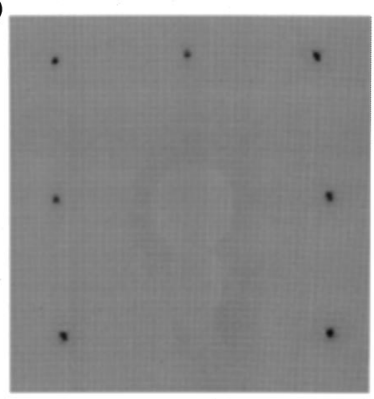

FIG. 2. The LEED patterns of the clean $\mathrm{Cu}_{3} \mathrm{Au}(100)$ surface $[($ a) $E=139$ $\mathrm{eV}]$ and a $9 \mathrm{ML} \mathrm{Ni}_{32} \mathrm{Pd}_{68}$ film $[(\mathrm{b}) E=135 \mathrm{eV}]$.

with $52 \%$ and $65 \% \mathrm{Pd}$ content to that of a pure Ni film. The intensity variations of the $\{00\}$ beam in MEED indicate a complicated growth mode for $\mathrm{Ni}$, but a quasi layer-by-layer (1.b.1.) growth for the alloy film improving with the Pd concentration. A feature common to all curves is the missing intensity maximum for the first monolayer (ML). According to extensive STM investigations this is due to an initial bilayer growth mode which switches to a layer-by-layer growth after completion of the second monolayer. ${ }^{11}$ For pure Ni films this 1.b.l. growth mode gradually turns over into an island growth which causes the damping of the intensity oscillations beyond the fifth layer. The resulting surface morphology is similar to the mesa-type structures found in $\mathrm{Ni} / \mathrm{Cu}(100){ }^{12}$ This behavior changes with the incorporation of $\mathrm{Pd}$. For $52 \% \mathrm{Pd}$ the amplitude of the oscillations is significantly higher than in the pure $\mathrm{Ni}$ case and less strongly damped, already indicating a pronounced tendency towards 1.b.l. growth. At $65 \% \mathrm{Pd}$ the 1.b.l growth is dominant up to coverages of more than $15 \mathrm{ML}$.

The reason for this pecular growth behavior may be attributed to the lattice misfit in the system. The misfit between $\mathrm{Ni}, \mathrm{Pd}$, and the $\mathrm{Cu}_{3} \mathrm{Au}$ lattice along the (100) direction is $\eta \approx+6 \%$ and $\eta \approx-4 \%$, respectively. By increasing the $\mathrm{Pd}$ content in the alloy the misfit is therefore reduced. Eventually an almost perfect lattice match can be achieved at $\mathrm{Pd}$ concentrations between $50 \%$ and $70 \%$.

We briefly comment on the structure of the films as seen by low energy electron diffraction (LEED). Figure 2 displays representative LEED patterns from the clean $\mathrm{Cu}_{3} \mathrm{Au}(100)$ surface and a NiPd film. The template surface is characterized by a $c(2 \times 2)$ superstructure resulting from the chemically ordered state. ${ }^{13}$ This superstructure vanishes upon deposition of $\mathrm{NiPd}$ and is replaced by a simple $p(1 \times 1)$ pattern. From the absence of any superstructure we must conclude that our $\mathrm{Ni}_{x} \mathrm{Pd}_{1-x}$ films are chemically disordered. On the basis of our present data, however, we cannot rule out the possibility of a partial local order. A preliminary analysis of the LEED I(V) curves obtained from these films is consistent with a face centered tetragonal (fct) structure, the tetragonal distortion of which changes with the $\mathrm{Pd}$ concentration. ${ }^{11}$ A more detailed picture of the atomic arrangement at the surface and the exact amount of the tetragonal distortion must involve dynamical LEED calculations.

The main objective of our work was the magnetic properties of the system $\mathrm{Ni}_{x} \mathrm{Pd}_{1-x} / \mathrm{Cu}_{3} \mathrm{Au}(100)$. These were

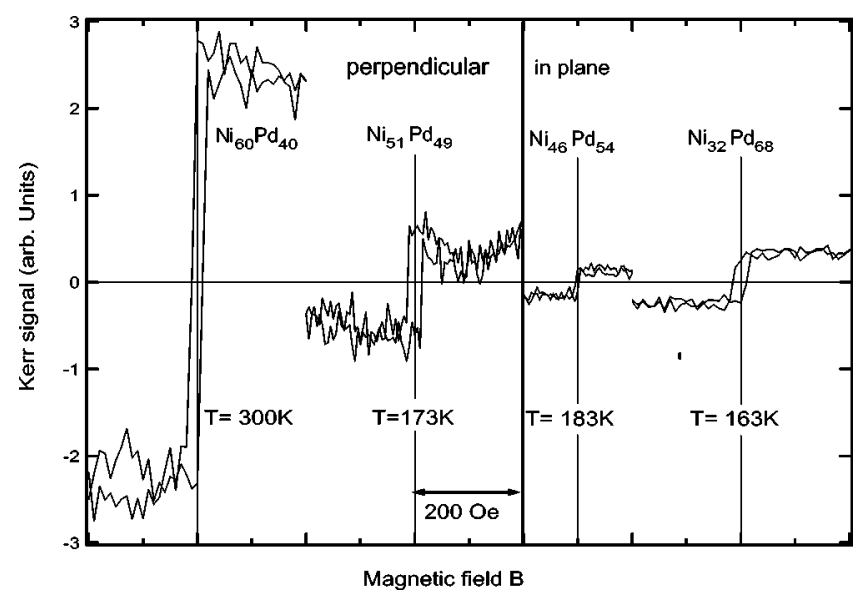

FIG. 3. The MOKE hysteresis loops of 9 ML NiPd films with varying Pd content showing the inverse spin reorientation transition at around $x$ $\approx 0.55$.

measured by magneto-optic Kerr effect (MOKE) in both the longitudinal and polar geometry. We find the films to be long-range ferromagnetically ordered up to $82 \% \mathrm{Pd}$, which was the highest Pd concentration investigated in our experiments so far. MOKE hysteresis loops from 9 ML thick films covering the compositions between $0.9 \geqslant x \geqslant 0.2$ are compiled in Fig. 3. The data show an in-plane remanent magnetic signal for $0.5 \geqslant x \geqslant 0.2$ and an out-of-plane magnetization for $1 \geqslant x \geqslant 0.5$. In other words, a spin-reorientation transition occurs with a variation of the Pd content in the alloy film. This inverse SRT is similar to the one observed for pure Ni films. We note, however, that the position of the SRT in the $9 \mathrm{ML}$ film is shifted on the thickness scale as compared to $\mathrm{Ni} / \mathrm{Cu}_{3} \mathrm{Au}(100)$ where it occurs at around $7 \mathrm{ML}$. Both the $\mathrm{Ni} / \mathrm{Cu}_{3} \mathrm{Au}(100)$ and $\mathrm{Ni} / \mathrm{Cu}(100)$ systems also show spin reorientation occurring as a function of temperature. ${ }^{10,14}$ As has already been discussed in theory, the influence of the temperature on the inverse SRT may be even stronger than on the regular SRT. ${ }^{15}$ For the case of $\mathrm{Ni}_{x} \mathrm{Pd}_{1-x} / \mathrm{Cu}_{3} \mathrm{Au}(100)$ this issue will be discussed elsewhere. ${ }^{11}$

In order to study a possible dependence of the SRT on the Pd concentration in more detail, we have varied both film thickness and composition. The resulting data are condensed a phase diagram (Fig. 4). We clearly see that the SRT is shifted continuously towards higher coverages with increasing Pd concentration. This is the first time that a composition dependent inverse SRT has been observed in ultrathin alloy films

The peculiar magnetic behavior reflected in Fig. 4 can be qualitatively understood by taking into account the strain dependence of the magnetization direction in Ni. The inverse SRT observed in pure Ni films is believed to occur because there is a strong bulklike magnetoelastic contribution $E_{\text {m.e. }}$ to the magnetic anisotropy energy caused by the lattice mismatch and the resulting tetragonal distortion in the film. This magnetoelastic contribution overcompensates the demagnetizing field $\left(E_{\text {demag }}\right)$ at a certain critical thickness $t_{\mathrm{SRT}}$, thereby leading to a flip of the magnetization into the direc- 


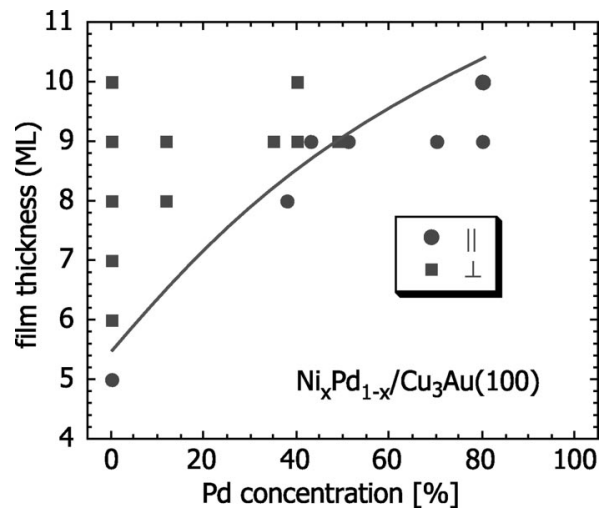

FIG. 4. Magnetic phase diagram of the $\mathrm{Ni}_{x} \mathrm{Pd}_{1-x}$ films. The line separating the regimes of in-plane and perpendicular magnetization serves as a guide to the eye only.

tion perpendicular to the film plane. ${ }^{9}$ At the thickness $t_{\mathrm{SRT}}$ we thus have the situation

$$
E_{\text {m.e. }}+E_{\text {crys }}=E_{\text {demag }}
$$

where $E_{\text {crys }}$ summarizes all magnetocrystalline anisotropy contributions.

In a first approximation we may assume a similar situation to hold for the alloy films as well. As discussed above in the context of growth, the incorporation of Pd in the Ni lattice leads to a reduction of the lattice mismatch and thus a strain relaxation in the film. As a consequence, also the magnetoelastic contribution will be reduced, thereby causing the SRT to shift towards higher film thicknesses, just as is observed in our experiment. We note that the actual strain dependence of the magnetoelastic anisotropy contribution $E_{\text {m.e. }}$ must be even larger than it appears from our data, because the saturation magnetization and thus the demagnetizating field $E_{\text {demag }}$ are also reduced with the Pd content. This must be taken into account in a quantitative analysis of the results. This quantitative analysis, however, is complicated by the fact that the magnetoelastic constants of the alloy films are not known. It is reasonable to assume that the magnetoelastic constants vary smoothly with the Pd concentration and that the Ni values may be used to describe the situation in films with small Pd content. For higher Pd concentrations the situation will be different, since Pd is a material with a significantly higher spin-orbit coupling than Ni. Further experimental and theoretical studies will be necessary to clarify this point.

In conclusion, we have demonstrated that good quality epitaxial $\mathrm{Ni}_{x} \mathrm{Pd}_{1-x}$ alloy films can be grown on $\mathrm{Cu}_{3} \mathrm{Au}(100)$. Both the growth and the magnetic properties show a pronounced influence of the film strain which may be varied by the Pd content. For the first time a compositiondependent inverse spin reorientation transition is observed in ultrathin alloy films.

\section{ACKNOWLEDGMENT}

This work was supported by Grant No. 05 SC8BDA 2 from the German Ministery of Education and Research (BMBF).

${ }^{1}$ See, for example, the contributions of J. G. Booth and K. H. J. Buschow in Ferromagnetic Materials Vol. 4, edited by E. P. Wohlfarth and K. H. J. Buschow (Elsevier, Amsterdam, 1988).

${ }^{2}$ G. J. Mankey et al., J. Vac. Sci. Technol. A 13, 1531 (1995).

${ }^{3}$ J. W. Freeland et al., Phys. Rev. B 57, 80 (1997).

${ }^{4}$ F. O. Schumann et al., Phys. Rev. Lett. 79, 5166 (1997).

${ }^{5}$ F. O. Schumann et al., Phys. Rev. B 56, 2668 (1997).

${ }^{6}$ A. Dittschar, M. Zharnikov, W. Kuch, C. M. Schneider, and J. Kirschner, Phys. Rev. B 57, R3209 (1998).

${ }^{7}$ G. R. Harp et al., Phys. Rev. Lett. 71, 2493 (1993).

${ }^{8}$ W. A. Ferrando, R. Segnan, and A. I. Schindler, Phys. Rev. B 5, 4657 (1972).

${ }^{9}$ B. Schulz and K. Baberschke, Phys. Rev. B 50, 13467 (1994).

${ }^{10}$ A. Braun, B. Feldmann, and M. Wuttig, J. Magn. Magn. Mater. 171, 16 (1997).

${ }^{11}$ M. Seider, U. Muschiol, R. Kaltofen, and C. M. Schneider (unpublished)

${ }^{12}$ J. Shen, J. Giergiel, and J. Kirschner, Phys. Rev. B 52, 8454 (1995).

${ }^{13}$ H. Okamoto, D. J. Chakrabarti, D. E. Laughlin, and T. B. Massalski, Bull. Alloy Phase Diagrams 8, 454 (1987).

${ }^{14}$ M. Farle, W. Platow, A. N. Anisiimov, P. Poulopoulos, and K. Baberschke, Phys. Rev. B 56, 5100 (1997).

${ }^{15}$ A. Hucht and K. D. Usadel, Phys. Rev. B 55, 12309 (1997). 